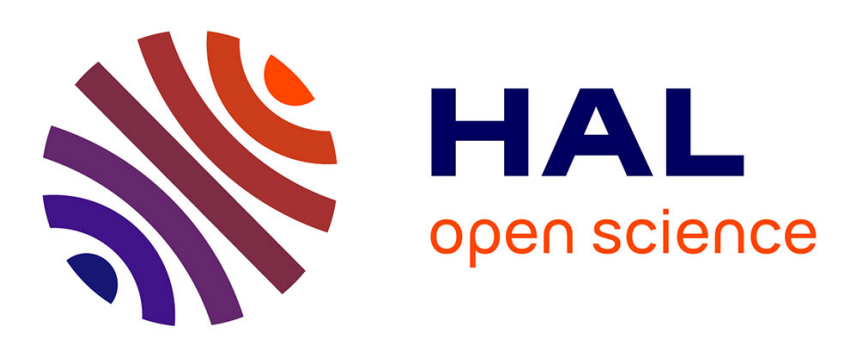

\title{
Assessing The Relevance Of Neurophysiological Patterns To Predict Motor Imagery-based BCI Users' Performance
}

\author{
Eidan Tzdaka, Camille Benaroch, Camille Jeunet, Fabien Lotte
}

\section{- To cite this version:}

Eidan Tzdaka, Camille Benaroch, Camille Jeunet, Fabien Lotte. Assessing The Relevance Of Neurophysiological Patterns To Predict Motor Imagery-based BCI Users' Performance. IEEE SMC 2020 - IEEE International conference on Systems, Man and Cybernetics, Oct 2020, Toronto / Virtual, Canada. hal-02971802

\section{HAL Id: hal-02971802 \\ https://hal.inria.fr/hal-02971802}

Submitted on 19 Oct 2020

HAL is a multi-disciplinary open access archive for the deposit and dissemination of scientific research documents, whether they are published or not. The documents may come from teaching and research institutions in France or abroad, or from public or private research centers.
L'archive ouverte pluridisciplinaire HAL, est destinée au dépôt et à la diffusion de documents scientifiques de niveau recherche, publiés ou non, émanant des établissements d'enseignement et de recherche français ou étrangers, des laboratoires publics ou privés. 


\section{Assessing The Relevance Of Neurophysiological Patterns To Predict Motor Imagery-based BCI Users' Performance}

\author{
Eidan Tzdaka \\ Inria Bordeaux Sud-Ouest \\ Talence, France, eidantz@gmail.com \\ Camille Jeunet
}

CLLE Lab, CNRS, Univ. Toulouse Jean Jaurès

Toulouse, France, camille.jeunet@univ-tlse2.fr

\author{
Camille Benaroch \\ Inria / LaBRI (Univ. Bordeaux, CNRS, Bordeaux INP) \\ Talence, France, camille.benaroch@inria.fr \\ Fabien Lotte \\ Inria / LaBRI (Univ. Bordeaux, CNRS, Bordeaux INP) \\ Talence, France, fabien.lotte@inria.fr
}

\begin{abstract}
Motor Imagery-based Brain-Computer Interfaces (MI-BCI) allow users to control a computer for various applications using their brain activity alone, which is usually recorded by an electroencephalogram (EEG). Although BCI applications are numerous, their use outside laboratories is still scarce due to their poor accuracy. Some users cannot use BCIs, a phenomenon sometimes called "BCI illiteracy", which impacts around $10 \%$ to $30 \%$ of BCI users, who cannot produce discriminable EEG patterns. By performing neurophysiological analyses, and notably by identifying neurophysiological predictors of BCI performance, we may understand this phenomenon and its causes better. In turn, this may also help us to better understand and thus possibly improve, BCI user training. Therefore, this paper presents statistical models dedicated to the prediction of MI-BCI user performance, based on neurophysiological users' features extracted from a two minute EEG recording of a "relax with eyes open" condition. We consider data from 56 subjects that were recorded in a 'relax with eyes open' condition before performing a MI-BCI experiment. We used machine learning regression algorithm with leave-onesubject-out cross-validation to build our model of prediction. We also computed different correlations between those features (neurophysiological predictors) and users' MI-BCI performances. Our results suggest such models could predict user performances significantly better than chance $(p \leq 0.01)$ but with a relatively high mean absolute error of $\mathbf{1 2 . 4 3 \%}$. We also found significant correlations between a few of our features and the performance, including the previously explored $\mu$-band predictor, as well as a new one proposed here: the $\mu$-peak location variability. These results are thus encouraging to better understand and predict BCI illiteracy. However, they also require further improvements in order to obtain more reliable predictions.
\end{abstract}

Index Terms-Brain-Computer Interfaces (BCI), electroencephalography (EEG), neurophysiological predictor

\section{INTRODUCTION}

Motor Imagery-based Brain-Computer Interfaces (MI-BCIs) allow users to control computers by producing brain activity patterns that are usually measured with Electroencephalography (EEG) [1]. MI leads to specific rhythmic macroscopic EEG oscillations (mostly in the $\mu$ and $\beta$ bands) in sensorimotor cortices, which can be detected using a BCI [1]. Thus, by discriminating MI patterns, the recognized patterns can be associated with a specific command for the computer [2], e.g., imagining feet movements to make a cursor move towards the bottom of the screen. Although BCI applications are numerous, e.g., for assistive technologies or entertainment, [3] [4], their use outside laboratories is rather scarce due to their poor accuracy. On average, one out of four or five user commands is misinterpreted by BCI systems based on 2 MI classes [5]. The BCI community tried to overcome this problem in different ways such as making users train longer a given MI task, to become more BCI proficient. The larger amount of data collected this way can also be used to refine BCI classifiers. There has also been attempts to change the instructions given or to improve EEG signal processing methods and hardware (e.g., electrodes) [6]. However, we also need to consider the user, who is one of the main components in the BCI loop, and may not be able to produce reliable EEG patterns, which is sometimes called "BCI illiteracy/deficiency". Producing distinct EEG patterns is a skill that must be mastered by the user [8], [9]. Therefore, user training is also essential and should be studied and improved so that users can become proficient at BCI control. Indeed, if a naive BCI user were unable to perform the desired MI commands due to a lack of ability to produce discriminable EEG patterns, regardless of the signal processing used, the BCI system would struggle and would issue random commands. Insufficient user's attention to the imagery tasks, or frustration due to incorrect feedback may also cause poor performances [5]. While the approaches presented above have only been partly successful, they could make BCIs work for some previously "illiterate" users [10]. However, some users still remain unable to use MI-BCI systems or have poor control.

There was thus some research work dedicated to finding predictors of BCI performance, in order to quickly identify whether a BCI user is likely to be able to control a given

\footnotetext{
${ }^{1}$ There is valid criticism about the broad use of the term "BCI illiteracy". Indeed, this concept seems to rely on an inaccurate presumption that users and their traits are the sole responsible for poor BCI performances [7].
} 
BCI [9], [11], [12]. Those studies showed that users' gender [13], daily hand movement [14], experience with state-of-theart technology [15] and good spatial abilities [9] are all related to user's MI-BCI performance.

These studies have explored users' traits as predictors, but not their neurophysiological correlates, nor other neurophysiological predictors. A different study [11] uncovered the $\mu$ band predictor, which can be determined from a two-minute recording of a 'relax with eyes open' condition. This predictor had a positive correlation with users' MI-BCI performance. In [12], the task of relaxing with open eyes was used again. They found out that high theta and low alpha EEG patterns could be observed for users with difficulty in using the BCI. Those works identified predictors correlated to BCI users performances. However, how well can they really predict the performances of new unseen users? Can we combine multiple predictors together to improve this prediction? We aim to answer these questions in this paper. In particular, its main objective is to create a computational model that could predict the performances of a BCI user based on multiple users' neurophysiological features. Thus, we first needed to identify predictors that correlate with MI-BCI performances. Our database contains 56 healthy MI-BCI naive participants who were asked to 'relax with eyes open' as a baseline for 3-minute followed by six 7-minute runs during which they had to learn to perform two MI-tasks, i.e., left and right-hand movement imagination. We used an elastic net regularized regression to predict between-subject MI-BCI performance variability. We extracted predictors based on the EEG power spectral density to build those models. We notably re-used the $\mu$-band predictor combined with other new spectral predictors proposed here (e.g., the width of the peak of the $\mu$-band) to create multivariate models to predict MI-BCI performances.

This paper is organized as follows: Section II describes the data set that we used and its paradigm, together with the predictor features and the approach used to build models and the elastic net regression. Then, Section III is dedicated to the results while Sections IV and V discuss the results and conclude the paper, respectively.

\section{Materials And Methods}

\section{A. Data set}

We used the data of fifty-six subjects $(\mathrm{N}=56)$ out of the fiftynine subjects from the experiment in [16] (29 women; age 1959; $\bar{X}=29 ; \mathrm{SD}=9.318$ ). We discarded three subjects for which the $\mu$-predictor (see the $\mu$-band predictor section) could not be computed due to noisy data: the optimization process failed to converge (note that we used the original authors' code). The main goal of this previous experiment was to assess the impact of the interaction between experimenters' and participants' gender on MI-BCI performances. Each subject participated in one MI-BCI session of 2 hours, comprising a three-minute recording of a 'relax with eyes open' condition followed by six 7-minute blocks (a.k.a runs) of trials in which the subject had to execute MI-tasks, i.e., imagine right or left-hand movements ( 1 of the 56 subjects did only four runs due to time limits).
Every subject performed 40 trials in a block, 20 per MI-task that appeared in random order. Each trial lasted for $8 \mathrm{~s}$. At $\mathrm{t}=0 \mathrm{~s}$ a cross was displayed on the screen. After two seconds the subject was provided with an acoustic signal which indicated the appearance of a red arrow, that appeared one second later. The red arrow direction indicated the type of MI-task to perform, e.g., a left pointing arrow to indicate the imagination of a movement of the left hand. From $t=4.250 \mathrm{~s}$ a blue bar was displayed as feedback, representing the classifier output: its length changed according to the classifier output and it appeared only if the instruction matched the recognized task (positive feedback only). The feedback lasted 3.75s.Afterwards the screen turns black and the user had a few seconds to rest before the next trial started.

\section{B. EEG Recordings and Signal Processing}

To record the EEG signals, 27 active scalp electrodes, referenced to the left ear, were used $(\mathrm{Fz}, \mathrm{FCz}, \mathrm{Cz}, \mathrm{CPz}, \mathrm{Pz}$, $\mathrm{C} 1, \mathrm{C} 3, \mathrm{C} 5, \mathrm{C} 2, \mathrm{C} 4, \mathrm{C} 6, \mathrm{~F} 4, \mathrm{FC} 2, \mathrm{FC} 4, \mathrm{FC} 6, \mathrm{CP} 2, \mathrm{CP} 4$, CP6, P4, F3, FC1, FC3, FC5, CP1, CP3, CP5, P3, 10-20 system). EEG signals were measured using a g.USBAmp (g.tec, Austria), sampled at $256 \mathrm{~Hz}$ and processed online using OpenviBE 2.1.0. In order to discriminate MI tasks from EEG data, participant-specific spectral and spatial filters have been used based on the two first runs (calibration runs). First, a participant-specific discriminant frequency band was selected using the heuristic algorithm proposed in [17]. It selects the frequency band whose power in the sensorimotor channels maximally correlates with the class labels. In this study, channels C3 and C4 were used after spatial filtering with a Laplacian filter. The discriminant frequency band was selected within the interval from $5 \mathrm{~Hz}$ to $35 \mathrm{~Hz}$, with $0.5 \mathrm{~Hz}$ large bins. Then EEG was filtered in that selected band with a Butterworth filter of order 5. To build the classifier, the Common Spatial Pattern (CSP) algorithm [17] was first used to optimize 3 pairs of spatial filters. CSP maximizes the signal band power difference between the two MI conditions. The log-transformed band power of the CSP filtered signals was used as input of a Linear Discriminant Analysis classifier.

\section{C. $\mu$-band predictor}

As predictor, we first computed the $\mu$-band predictor proposed in [11]. We first used the raw EEG data from the three minutes recordings in a 'relax with eyes open' condition, and used band-pass filtering between $2 \mathrm{~Hz}$ to $40 \mathrm{~Hz}$ to reduce physiological noise and power line noise that might affect the EEG recording. We then created two Laplacian channels around $\mathrm{C} 3$ and $\mathrm{C} 4$, from 5 monopolar channels for each. From the three minutes of recording, we only used the middle two minutes to avoid user movement at the beginning and end of the recording. From these data, we computed the power spectral density (PSD) between $2 \mathrm{~Hz}$ to $30 \mathrm{~Hz}$ by using Welch's method with a Fast Fourier transform (FFT) size of 4096. The PSD were computed for each of the two Laplacian channels. 
As proposed in [11], we modeled the PSD curve by using the sum of two functions:

$$
\begin{gathered}
g(f ; \lambda, \mu, \sigma, \mathbf{k})=g_{1}(f ; \lambda, k)+g_{2}(f ; \mu, \sigma, k) \\
g_{1}(f ; \lambda, \mathbf{k})=k_{1}+\frac{k_{2}}{f^{\lambda}} \\
g_{2}(f ; \mu, \sigma, \mathbf{k})=k_{3} \varphi\left(f ; \mu_{1}, \sigma_{1}\right)+k_{4} \varphi\left(f ; \mu_{2}, \sigma_{2}\right)
\end{gathered}
$$

where $\mathbf{k}=\left(k_{1}, k_{2}, k_{3}, k_{4}\right) \in \mathbb{R}^{4}, \lambda \in \mathbb{R}$ and $\varphi(\cdot ; m, s)$ indicates the probability density function of a normal distribution with mean $m$ and standard deviation $s$. Function $g_{1}$ models the noise spectrum and $g_{2}$ models the peaks around the $\mu$ (8$13 \mathrm{~Hz})$ and $\beta(13-30 \mathrm{~Hz})$ bands. Therefore $\mu_{1}$ and $\mu_{2}$ represent the $\mu$ and $\beta$ peaks locations, $\sigma_{1}$ and $\sigma_{2}$ their scale and $k_{1}$ and $k_{2}$ their amplitude. The nine parameters are estimated by minimizing the $L_{2}$-norm of the difference vector $P S D(\mathbf{f})$ $g(\mathbf{f} ; \lambda, \mu, \sigma, \mathbf{k})$, where $\mathbf{f}$ is the vector of all available frequency values for the PSD. The $\mu$-band predictor is defined as $\max _{f \in \mathbf{f}} g_{2}(\mathbf{f} ; \mu, \sigma, \mathbf{k})-g_{1}(\mathbf{f} ; \lambda, \mathbf{k})$. Here, we force $\mathbf{f}$ to be in the range of the $\mu$ band frequencies, which means that we search for the maximum difference between $g_{2}$ to $g_{1}$ only between $5 \mathrm{~Hz}$ to $15 \mathrm{~Hz}$.

\section{Variable to predict}

In the experiment, MI-BCI performance was assessed in terms of mean classification accuracy, i.e., the mean accuracy measured over all the windows of the feedback periods of the runs 3 to 6 (online feedback runs). We tried to predict each subject performance (i.e., their classification accuracy) using an elastic net model with features extracted from the 2-minute baseline of that subject.

\section{E. Predictor Variables}

In our study, we extracted features from the PSD curve of each subject during the recording of the baseline. These features describe the PSD properties, and are used as input of the model to predict the performance. As described in Figure 1, the features we extracted are the following:

- $\boldsymbol{\mu}$-band predictor that was computed by: $\max _{f \in \mathbf{f}} g_{2}(\mathbf{f} ; \mu, \sigma, \mathbf{k})-g_{1}(\mathbf{f} ; \lambda, \mathbf{k})$, where $\mathbf{f}$ is in the $\mu$-band frequency range (5 to $15 \mathrm{~Hz}$ ).

- $\beta$-band predictor computed similarly to the $\mu$-band predictor, as $\max _{f \in \mathbf{f}} g_{2}(\mathbf{f} ; \mu, \sigma, \mathbf{k})-g_{1}(\mathbf{f} ; \lambda, \mathbf{k})$, where $\mathbf{f}$ is in the $\beta$-band frequency range ( 15 to $25 \mathrm{~Hz}$ ).

- Log transformation of $\mu$-band predictor and log transformation of $\beta$-band predictor to decrease the variability of the data and make it more normally distributed.

- Frequency of the $\mu$-band peak and frequency of the $\beta$-band peak.

- Width of the $\boldsymbol{\mu}$-band peak and width of the $\boldsymbol{\beta}$-band peak first calculated by evaluating the height $h_{\text {eval }}$ with $h_{\text {eval }}=h_{\text {Peak }}-P$ where $h_{P e a k}$ is the real height of the peak and $P$ is the peak prominence, [18]. Then an horizontal line is drawn to evaluate the height of both sides of the peak bases. Starting at the peak current vertical position until the lines either intersect a slope,

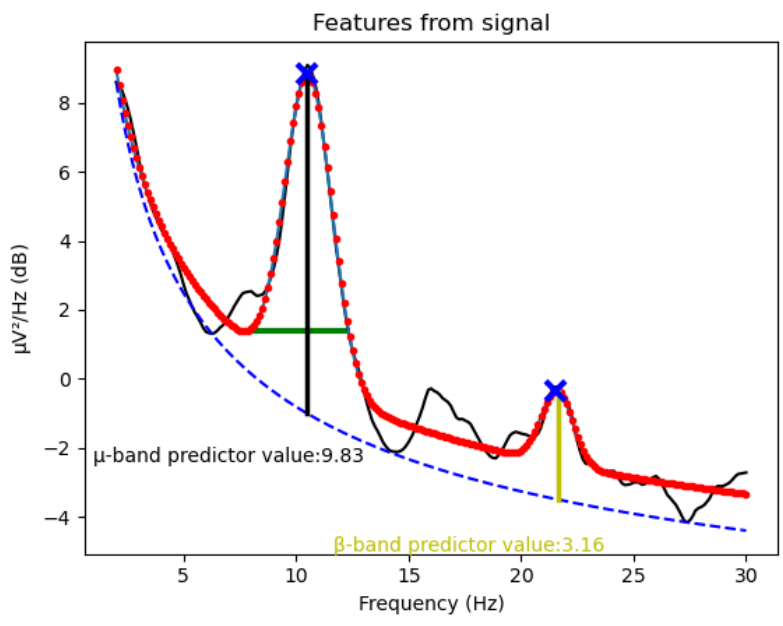

Fig. 1. In the plot, we can see most of the features that were extracted from the signal. The solid black signal is the original power spectral density of the user, the dashed blue signal is the estimated floor noise $\left(g_{1}(f ; \lambda, \mathbf{k})\right)$ while the dotted red signal is the fitted values $g(f ; \lambda, \mu, \sigma, \mathbf{k})$. The vertical green line represent the base of the peaks (and the width of the peak for the $\mu$-band peak). The two horizontal lines describe the predictors, the black one is for $\mu$-band predictor and the yellow one is for the $\beta$-band predictor. The blue ' $\mathrm{X}$ ' represents the frequency of the peak and the number of peaks in the signal i.e., 2 in this example.

the signal border or crosses the vertical position of the peak base. The width is the distance between the two chosen points.

- Variance of the $\mu$-band amplitudes across the frequencies comprised in the peak width.

- Variance of the $\mu$-band frequencies comprised in the peak width.

- Number of peaks, i.e., the amount of distinct peaks in the signal which is either 0,1 or 2 .

Furthermore, we also investigated how the $\mu$ peak varies across time. Indeed, its variability may explain differences in mean peak amplitude or could be related to BCI performances. For that, we make use of the whole three minutes baseline recording, and estimated the variance of the peak location, width, and amplitude across all 10s epochs with 3 s overlap from that baseline. This led to three additional features:

- Variance of the frequency of the $\mu$-band peak across time: we calculated the frequency of the $\mu$-band peak for each epoch and calculated the variance of those values.

- Variance of the width of the $\boldsymbol{\mu}$-band peak across time: we calculated the width of the $\mu$-band peak for each epoch and calculated the variance of those values.

- Variance of the amplitude of the $\mu$-band peak across time: we calculated the amplitude of the $\mu$-band peak for each epoch and calculated the variance of those values.

If no significant peak was found in the given epoch, all three values that were mentioned above were set to 0 for that epoch, in order to indicate the lack of peak in the $\mu$-band.

These features were extracted from both Laplacian channels $\mathrm{C} 3$ and $\mathrm{C} 4$, leading to a total of 22 features per subject. They 
were then used by our model to predict MI-BCI performances. It should be noted that except the $\mu$-band predictor, all other features were explored for the first time in this paper.

\section{F. Correlation analyses}

We performed Pearson correlation analyses for each feature, to estimate how well they were correlated to performances. As we performed numerous tests, we performed a correction for multiple testing using the Benjamini-Hochberg $(\mathrm{BH})$ procedure [19] to control the False Discovery Rate (FDR).

\section{G. Analyses}

We first normalized each feature by using Z-score. Then we built our Elastic Net model and compared its predictions to that of a random model to investigate its reliability.

1) Elastic Net: We used Elastic Net regression in order to get an interpretable model that was able to predict MI-BCI users' performance. Elastic Net regression uses $l 1$ and $l 2$-norm regularization with two penalty parameters $\lambda_{1}$ and $\lambda_{2}$ [20]. This combination allows us to create a sparse model with some weights that are non-zero like Lasso while still maintaining the regularization properties of Ridge regression [20]. Elastic Net is also more robust and stable than Lasso [20] since with Lasso variable selection can be too dependent on data and thus unstable. In addition, Elastic Net can handle correlated variables better than Lasso. Indeed, with such variable, Lasso usually randomly chooses one and ignores the others, i.e., one of the coefficients of the correlated features will be nonzero while the others will be zeros. In contrast, Elastic Net will pick both of them with similar coefficient values. To set up the model we need: $y \in \mathbb{R}^{n}$ which here is the MIBCI performance vector, a matrix $X \in \mathbb{R}^{n \times p}$ with $p$ features (here the neurophysiological predictors) for $n$ subjects and a coefficient vector $\beta \in \mathbb{R}^{n}$ which is the regression weight. With Elastic Net, the regression weights are estimated by:

$$
\hat{\beta}_{e-n e t}=\underset{\beta \in \mathbb{R}^{p}}{\operatorname{argmax}}\|y-X \beta\|_{2}^{2}+\lambda_{1}\|\beta\|_{1}+\lambda_{2}\|\beta\|_{2}^{2}
$$

where, $\|u\|_{2}^{2}=\sum_{i=1}^{n} u^{i}$ for $u \in \mathbb{R}^{n},\|\beta\|_{1}=\sum_{j=1}^{p}\left|\beta_{j}\right|$ and $\|\beta\|_{2}^{2}=\sum_{j=1}^{p} \beta_{j}^{2}$. In order to determine the ratio between $\lambda_{1}$ and $\lambda_{2}$, we performed inner cross-validation (leave-onesubject-out cross-validation) on the $\mathrm{N}-2$ subjects (our 'inner' training set) to estimate the optimal ratio, i.e., the one that minimizes the mean absolute error on the training set. Then we used this optimal ratio to build the model for the N-1 training subjects. The left out subject is the test set. This process was repeated by using separately each subject as the test set.

2) Random model: The random model operates as an empirical chance level for our data. By rearranging the training set BCI performances randomly and keeping the features the same, we broke the before-known relationship between features and performance. We kept the same Elastic Net parameters $\left(\lambda_{1}\right.$ and $\left.\lambda_{2}\right)$ found in the inner cross-validation. Moreover, we kept the same features value and performance score for the test set that was left out to compare the actual model to the random one. We ran the Elastic Net model for

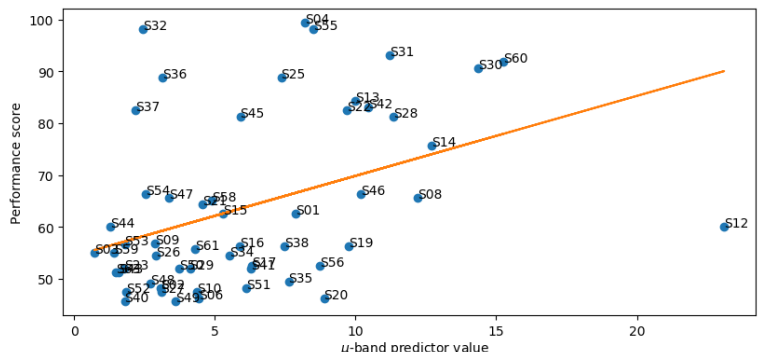

Fig. 2. Correlation of the $\mu$-band predictor with MI-BCI performance, each dot corresponds to one participant. The linear line is the least-squares polynomial fit of the $\mu$-band predictor and the corresponding MI-BCI performance.

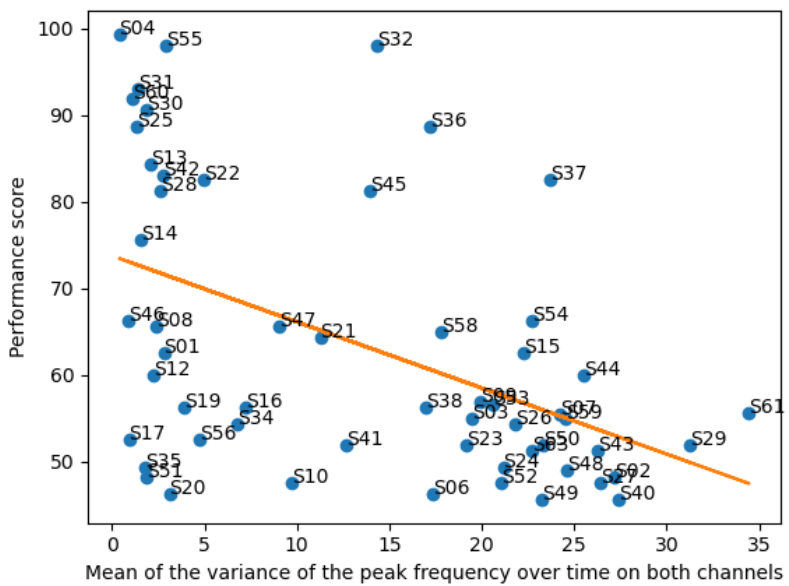

Fig. 3. Correlation of the mean of the variance of the peak frequency over time on both channels with MI-BCI performance, each dot corresponds to one participant. The linear line is the least-squares polynomial fit of the mean of the variance of the peak frequency over time on both channels and the corresponding MI-BCI performance.

the same test subject with the randomly shuffled training set. We did this process 10,000 times and stored the mean absolute error to obtain the distribution of the chance prediction performances. We then sorted the values in descending order and computed the $95^{\text {th }}$ and $99^{\text {th }}$ percentiles, which represent the chance level for the mean absolute error for $p=0.05$ and $p=0.01$ respectively.

\section{RESULTS}

\section{A. Correlation analyses}

We obtained a correlation of $r=0.426\left(p<0.01, p_{a d j} .<\right.$ 0.012 after BH procedure) (Figure 2) for the $\mu$-band predictor. We also found a correlation of $r=0.27(p<0.05)$ between the mean of the $\mu$ peak width over both channels and the performance. In other words, the larger the width of the peak the better the performance. However, after FDR correction, this correlation was not significant anymore $\left(p_{a d j} .<0.11\right)$. None of the $\beta$-band predictors were significantly correlated to performances though. We did find a correlation between the 
number of peaks in both channels and the performance $(r=$ $0.351, p<0.01, p_{a d j}$. $<0.032$ ). We also found a correlation between the mean (over channels) of the variance of the $\mu$ band peak frequency over time (Figure 3) and the performance $\left(r=-0.477, p<.0001, p_{a d j} .<.0001\right)$. This correlation seems mostly driven by the number of epochs with no peak, which is also negatively correlated to the performance $(r=$ $-0.42, p<0.001, p_{a d j}$. $\left.<0.003\right)$. Table I summarizes the results of all correlation analyses. Each feature is the mean of the values from both EEG channels.

TABLE I

CORRELATION RESULTS (BOLD P-VALUES ARE SIGNIFICANT. EACH FEATURE IS THE MEAN OF THE PREDICTOR VALUES FROM BOTH C3 AND C4 EEG CHANNELS.)

\begin{tabular}{|c|c|c|c|}
\hline Feature & $\boldsymbol{r}$ & $\boldsymbol{p}$-value & $\begin{array}{c}\boldsymbol{a} \text { adj } \\
\boldsymbol{p} \text {-value }\end{array}$ \\
\hline$\mu$-band predictor & 0.426 & $\mathbf{0 . 0 0 1}$ & $\mathbf{0 . 0 1 2}$ \\
\hline$\beta$-band predictor & 0.219 & 0.105 & 0.18 \\
\hline mean frequency of the $\mu$-band peak & -0.064 & 0.639 & 0.698 \\
\hline mean frequency of the $\beta$-band peak & 0.255 & 0.058 & 0.115 \\
\hline mean of peak width of the $\mu$ peak & 0.268 & $\mathbf{0 . 0 4 6}$ & 0.112 \\
\hline $\begin{array}{c}\text { mean of the variance } \\
\text { of the } \mu \text { peak amplitude }\end{array}$ & 0.045 & 0.743 & 0.743 \\
\hline $\begin{array}{c}\text { mean of the variance } \\
\text { of the } \mu \text { peak frequency }\end{array}$ & 0.178 & 0.19 & 0.28 \\
\hline $\begin{array}{c}\text { sum of the number of } \\
\text { peaks from both channels }\end{array}$ & 0.351 & $\mathbf{0 . 0 0 8}$ & $\mathbf{0 . 0 3 2}$ \\
\hline $\begin{array}{c}\text { mean of the variance of the } \mu \text {-band } \\
\text { peak frequency over time }\end{array}$ & -0.477 & $\mathbf{0 . 0 0 1}$ & $\mathbf{0 . 0 0 1}$ \\
\hline $\begin{array}{c}\text { mean of the variance of the } \mu \\
\text { peak amplitude over time }\end{array}$ & 0.144 & 0.299 & 0.359 \\
\hline $\begin{array}{c}\text { mean of the variance of the } \mu \\
\text { peak width over time }\end{array}$ & 0.26 & 0.058 & 0.115 \\
\hline \multicolumn{2}{|c|}{} \\
\hline
\end{tabular}

\section{B. Model}

After using Elastic Net regression and leave-one-subjectout cross-validation to create a model for each test user, we computed the mean absolute error across all the models. The results can be found in Figure 4. This mean error was $12.43 \%$. We compared it to the mean absolute error that we got from the 10,000 random models. The estimated chance level (from the random models) was $12.85 \%$ and $13.24 \%$ for $p=0.01$ and $p=0.05$ respectively. Therefore, on average, our prediction models were better than chance at $p<0.01$.

\section{DISCUSSION}

\section{A. $\mu$-band predictor}

From our results, we successfully reconfirmed the value of the $\mu$-band predictor [11] on our database, thus further emphasizing its relevance and usefulness. Since MI-BCI operates by recognizing event-related desynchronization (ERD) [21] performed voluntarily by the user, the higher the $\mu$ amplitude at rest, the more noticeable its decrease (i.e., an ERD) during the MI task. The $\mu$-band predictor represents the $\mu$ amplitude and thus, the bigger the $\mu$-band predictor, the better the chance that the user will be successfully gain control of the MI-BCI system.
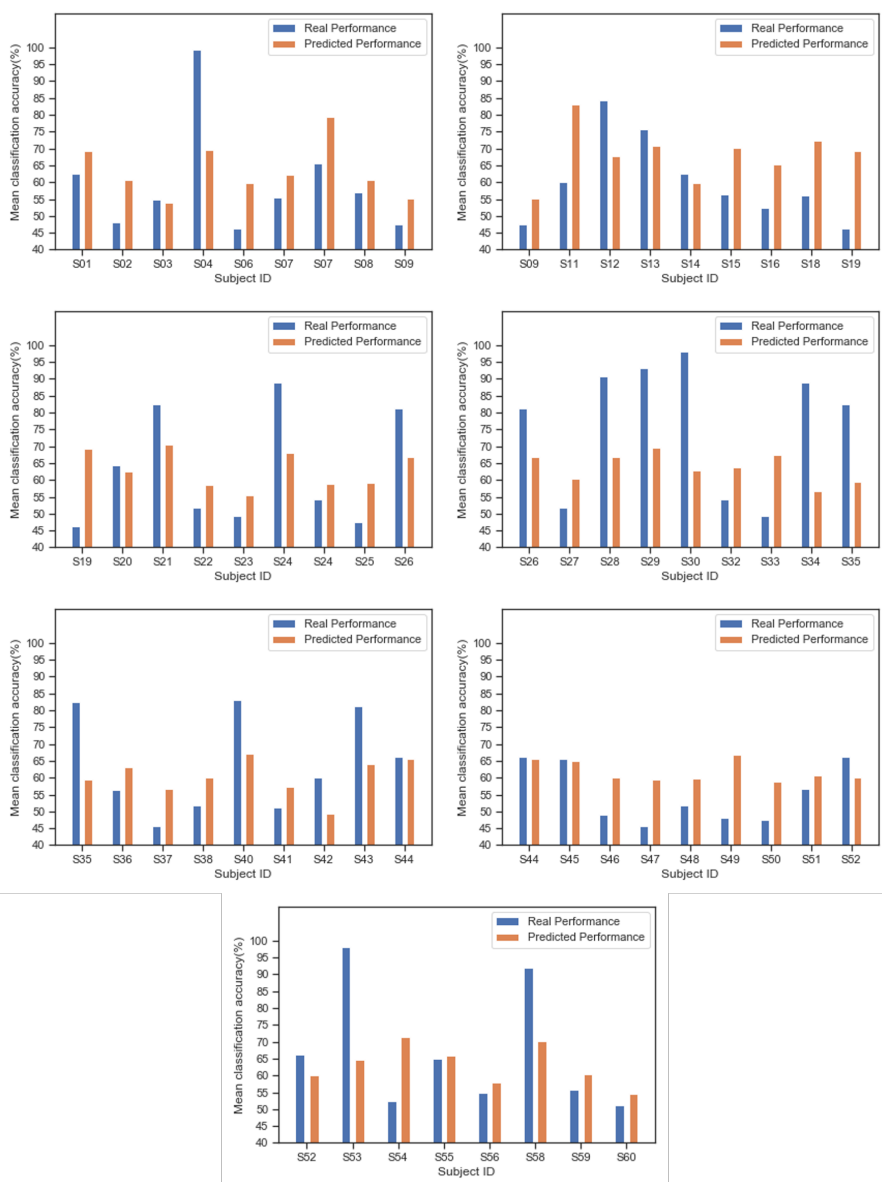

Fig. 4. Results of the Elastic net predictions compared to the real performance. In blue the real performance of each subject and in orange, the predicted performance of each subject generated using our model.

\section{B. Correlations for the new neurophysiological features}

In this paper, we also proposed and studied new neurophysiological features, some of which proving to be new significant predictors of performance. Among the significant correlations that were found, the mean width of the $\mu$ peak over both $\mathrm{C} 3$ and $\mathrm{C} 4$ channels was correlated to the users' performance. However, after FDR corrected its significance dropped under significance level which might suggest that it might not be a predictor of performance, or at least not a strong one. We did not to find significant $\beta$-band predictors. However, we did find a significant correlation between the number of peaks in both channels with the performance. In other words, our results suggest that MI-BCI users who have two distinct peaks (i.e., one $\mu$ and one $\beta$ peak) perform better than those who only have one peak. The $\beta$-band peak is known to be suppressed during MI (ERD) [21]. As MI-BCIs are based on these changes in the spectral power of the EEG signal in both $\mu$ and $\beta$, that might explain the correlation between the number of peaks and performances. Additionally, outside of $\mathrm{BCI}$, pre-training $\beta$-band ERD in the sensorimotor cortex significantly accounted for motor performance [22]. Moreover, pre-movement resting $\beta$-band power in the sensorimotor cor- 
tex was also significantly enhanced after training a motor task and performing it successfully [22]. Both observations might thus also explain why having two peaks, including a $\beta$ one, at rest could also explain motor BCI performances.

Finally, we found that the more stable the $\mu$-band peak (in terms of frequency location), the better the chance for the subject to perform well. A possible interpretation for that phenomenon could be that the more variable the $\mu$ location, the more its amplitude would be averaged out across time, thus making the detection of an ERD more difficult and lowering BCI performances. Alternatively, this $\mu$ peak variability might make it more difficult for machine learning algorithms to identify a clear and stable EEG pattern to use for classification. This would need to be studied in more details in the future. Altogether, these observed correlations should help us to understand and predict MI-BCI performance.

\section{Elastic net model}

Altogether, the Elastic Net, although significantly better than chance, had a relatively high mean absolute error. With more data we might obtain better predictions. Moreover, with more data, we might use a different cross-validation method which might lead to more accurate predictions. We do plan to add more features to our model, both neurophysiological predictors, e.g., before or during trials, and non-neurophysiological ones, e.g., traits [23]. The feedback paradigm that was used in the BCI experiment, although a standard one (which is why we studied it), is also known to be non-optimal [24]. This may explain why several of our subjects had below chance level performance. Perhaps due to the later, the best performers were underestimated by the models.

\section{Conclusions}

In this study, we used data of 56 subjects to predict their performances based on features we extracted from their EEG signal at rest. The objective was to create a machine learning model to predict the user BCI performance, and therefore better understand, and possibly improve BCI user training protocols. In order to achieve this goal, we used an Elastic Net that promotes sparse solutions. Such model obtained better than chance prediction performance. We hypothesize that by combining this model with other factors such as psychometric and personality questionnaires data, we might be able to create a more robust and precise model of prediction for MI-BCI performances. We also identified three different neurophysiological predictors that are significantly correlated to the users' BCI performance. Altogether, this study contributed to increase our understanding of BCI illiteracy/deficiency and paves the way for more advanced computational modeling of MI-BCI users performances. On the longer-term, such models should help to better understand and thus optimize BCI designs.

\section{ACKNOWLEDGMENTS}

This work was supported by the European Research Council with project BrainConquest (grant ERC-2016-STG-714567).

\section{REFERENCES}

[1] J. Wolpaw, N. Birbaumer, D. McFarland, G. Pfurtscheller, and T. Vaughan, "Brain-computer interfaces for communication and control," Clinical Neurophysiology, vol. 113, no. 6, pp. 767-791, 2002.

[2] G. Pfurtscheller, C. Neuper, D. Flotzinger, and M. Pregenzer, "EEGbased discrimination between imagination of right and left hand movement," EEG Clin. Neurophy., vol. 103, no. 6, pp. 642-651, 1997.

[3] A. Kübler, B. Kotchoubey, J. Kaiser, J. Wolpaw, and N. Birbaumer, "Brain-computer communication: Unlocking the locked in," Psychological Bulletin, vol. 127, no. 3, pp. 358-375, 2001.

[4] B. Allison, S. Dunne, R. Leeb, J. D. R. Millan, and A. Nijholt, Towards Practical Brain-Computer Interfaces. Berlin: Springer Berlin, 2014.

[5] C. Guger, G. Edlinger, W. Harkam, I. Niedermayer, and G. Pfurtscheller, "How many people are able to operate an EEG-based brain-computer interface (BCI)?” IEEE Trans. Neur. Syst. Rehab, vol. 11, no. 2, pp. 145-147, 2003.

[6] B. Allison and C. Neuper, "Could anyone use a BCI?" in BrainComputer Interfaces: Applying our Minds to Human-Computer Interaction, A. Nijholt and D. Tan, Eds. Springer London, 2010, pp. 35-54.

[7] M. C. Thompson, "Critiquing the concept of BCI illiteracy," Science and Engineering Ethics, vol. 25, no. 4, pp. 1217-1233, 2018.

[8] C. Neuper, R. Scherer, S. Wriessnegger, and G. Pfurtscheller, "Motor imagery and action observation: Modulation of sensorimotor brain rhythms during mental control of a brain-computer interface," Clinical Neurophysiology, vol. 120, no. 2, pp. 239-247, 2009.

[9] C. Jeunet, B. N'Kaoua, and F. Lotte, "Advances in user-training for mental-imagery-based BCI control: Psychological and cognitive factors and their neural correlates," in Progress in brain research. Elsevier, 2016, vol. 228, pp. 3-35.

[10] A. Nijholt and D. Tan, "Brain-computer interfacing for intelligent systems," IEEE Intelligent Systems, vol. 23, no. 3, pp. 72-79, 2008.

[11] B. Blankertz, C. Sannelli, S. Halder, E. M. Hammer, A. Kübler, K.-R. Müller, G. Curio, , and T. Dickhaus, "Neurophysiological predictor of SMR-based BCI performance," NeuroImage, vol. 51, no. 4, 2010.

[12] M. Ahn, H. Cho, S. Ahn, and S. Jun, "High theta and low alpha powers may be indicative of BCI-illiteracy in motor imagery," PLOS ONE, vol. 8, no. 11, p. e80886, 2013.

[13] A. Randolph, "Not all created equal: Individual-technology fit of braincomputer interfaces," in Hawaii Int. Conf. Syst. Sci., 2012.

[14] A. Randolph, M. Jackson, and S. Karmakar, "Individual characteristics and their effect on predicting mu rhythm modulation," International Journal of Human-Computer Interaction, vol. 27, no. 1, pp. 24-37, 2010.

[15] W. Burde and B. Blankertz, "Is the locus of control of reinforcement a predictor of brain-computer interface performance?" in Proc. Int. Graz BCI Conf, 2006, pp. 76-77.

[16] A. Roc, L. Pillette, B. N'Kaoua, and F. Lotte, "Would motor-imagery based BCI user training benefit from more women experimenters?" in Proc. Int. Graz BCI Conf., 2019.

[17] B. Blankertz, R. Tomioka, S. Lemm, M. Kawanabe, and K. Muller, "Optimizing spatial filters for robust EEG single-trial analysis," IEEE Signal Processing Magazine, vol. 25, no. 1, pp. 41-56, 2008.

[18] P. Virtanen et al., "SciPy 1.0: Fundamental Algorithms for Scientific Computing in Python," Nature Methods, vol. 17, pp. 261-272, 2020.

[19] Y. Benjamini and Y. Hochberg, "Controlling the false discovery rate: A practical and powerful approach to multiple testing," Journal of the Royal Statistical Society: Series B (Methodological), vol. 57, no. 1, pp. 289-300, 1995.

[20] H. Zou and T. Hastie, "Regularization and variable selection via the elastic net," Journal of the Royal Statistical Society, vol. 67, no. 2, pp. 301-320, 2005.

[21] G. Pfurtscheller and F. L. D. Silva, "Event-related eeg/meg synchronization and desynchronization: basic principles," Clinical Neurophysiology, vol. 110, no. 11, pp. 1842-1857, 1999.

[22] S. Espenhahn, B. C. van Wijk, H. E. Rossiter, A. O. de Berker, N. D. Redman, J. Rondina, J. Diedrichsen, and N. S. Ward, "Cortical beta oscillations are associated with motor performance following visuomotor learning," NeuroImage, vol. 195, pp. 340-353, 2019.

[23] C. Benaroch, C. Jeunet, and F. Lotte, "Are users' traits informative enough to predict/explain their mental-imagery based bci performances?" in Proc. Int. Graz BCI Conf., 2019.

[24] C. Jeunet, E. Jahanpour, and F. Lotte, "Why standard brain-computer interface (BCI) training protocols should be changed: an experimental study," Journal of Neural Engineering, vol. 13, no. 3, p. 036024, 2016. 